\title{
Intellectual Precise Temperature Sensor with Wireless Interface
}

\author{
M.Yu. Shtern*, Ya.S. Kozhevnikov, Yu.I. Shtern, R.E. Mironov, I.S. Karavaev \\ AND M.S. ROGACHEV \\ National Research University of Electronic Technology - MIET, \\ Bld. 1, Shokin Square, Zelenograd, Moscow, Russia, 124498
}

\begin{abstract}
Intellectual precise temperature sensor with wireless interface (ITWS) for contact measurements with absolute error of $\pm 0.05^{\circ} \mathrm{C}$ in the temperature range from 5 to $95^{\circ} \mathrm{C}$ was developed. Platinum thin film resistance thermometer was used as the sensitive unit. High accuracy of measurements is supported by following. Investigations and modeling of temperature dependence of resistivity for sensitive unit allowed to develop mathematical model ensuring calculation of temperature with the error not exceeding $5 \times 10^{-3}{ }^{\circ} \mathrm{C}$. Original patented design, and hardware and software solutions for ITWS were developed. Method and mathematical models for thermocompensation of electronic components of ITWS were elaborated, which allowed sufficient decrease of measurement error during measurements and exploitation. Methodology, and hardware and software measuring system for individual ITWS calibration in automatic regime were developed, which include correction of mathematical model for the calculation of temperature for each sensor. ITWS has several design and technological solutions, and is developed for the temperature measurements of heat transfer agent in the pipelines of heating and hot water supply systems, independently of pipeline diameter. Measured data are transferred by radio channel to the recording and display devices. ITWS are used in automated systems for energy carrier controlling and determination of individual heat consumption. ITWS consists of following modules: sensitive unit, analog-to-digital converter, microcontroller, radio transceiver (carrier frequency is 434 or $868 \mathrm{MHz}$, output power of transmitter is not more than $10 \mathrm{~mW}$ ), antenna and power supply $(3.6 \mathrm{~V})$.
\end{abstract}

DOI: 10.12693/APhysPolA.129.779

PACS/topics: 07.20.Dt

\section{Introduction}

Temperature is a vital thermodynamic parameter in determination of the amount of heat passed from the heating system to the environment. One of the main issues of heat consumption metering systems development is a lack of accuracy in temperature measurement. Of course there are a lot of thermometers which are even more accurate than necessary for the heat consumption metering systems, but their price is too high for use in every apartment. The accuracy of heat consumption metering is mainly determined by the accuracy of temperature measurement. Therefore, nowadays, it is necessary to develop new techniques and instruments for accurate temperature measurements in individual heat consumption metering systems [1].

The authors have proposed a new intellectual temperature sensor with wireless communication interface (ITWS) that is intended for contact measurement in the range of 5 to $95^{\circ} \mathrm{C}$ with an accuracy of $\pm 0.05^{\circ} \mathrm{C}$.

\section{Principle}

The structure of ITWS is shown in Fig. 1. ITWS consists of two functional units: an electronic thermometer and a transmitter, which are arranged on a single printed circuit board. The electronic thermometer

\footnotetext{
*corresponding author; e-mail: m.y.shtern@gmail.com
}

includes the following components: a thin film platinum resistive temperature detector (Pt-RTD1000) which is connected to the bridge circuit and a 16-bit ultralow-power microcontroller MSP430F2003 with a 16-Bit Sigma-Delta A/D. The transmitter consists of a microcontroller MSP430F2132, an RF transceiver CC1101 and an antenna. ITWS also includes a quartz crystal resonator with temperature stability of $10 \mathrm{ppm}$, a battery and a magnetically-operated sealed switch.

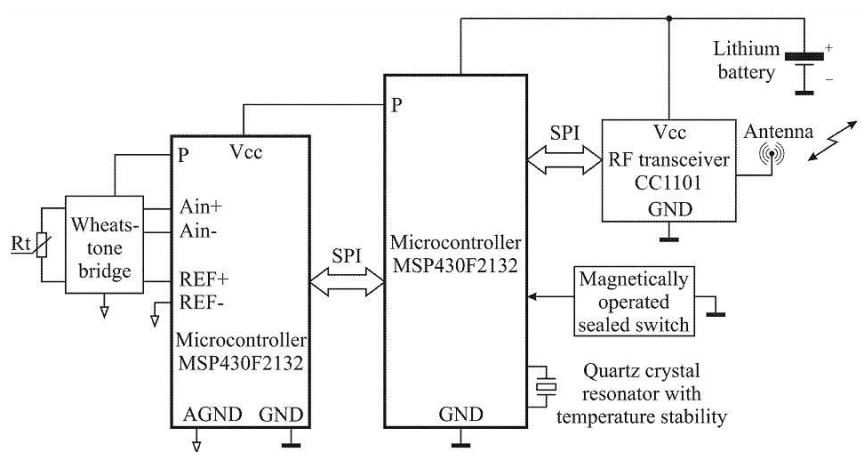

Fig. 1. The structure of ITWS.

Temperature measurement by ITWS is carried out as follows. A voltage drop on the Wheatstone bridge, one leg of which includes Pt-RTD1000, is measured by the MSP430F2003 microcontroller and then converted into the binary code. This data is transferred to the 
MSP430F2132 microcontroller over SPI where it is accumulated and stored in a buffer storage. From the buffer, the data is transmitted to the RF transceiver from where it is broadcast to a wireless repeater.

The transceiver conducts the following operations: hardware conversion of the binary coded packet data into the Manchester code, FSK modulation and amplification of the modulated signal. The receiver implements the following operations: frequency selection and amplification of the RF signal, demodulation of the FSK signal, and conversion of the Manchester code into the binary code.

The type of the radio exchange is synchronous-address with code division and control of channel occupancy. ITWS is powered by a lithium battery with a nominal voltage of $3.6 \mathrm{~V}$. The data from ITWS is wirelessly transmitted to a wireless repeater, and then via RS-485 to a computer, where proprietary software calculates temperature in accordance with a given transfer function and displays the results. The software installed on the PC also defines the ITWS operation algorithms, configures measurement regimes, accumulates and stores the measured data.

\section{The ITWS design}

The ITWS design is shown in Fig. 2. Functional units of ITWS are formed on a single printed circuit board $(\mathrm{PCB})$, which increases the reliability and facilitates the assembly. The PCB is enclosed into a housing with ventilation holes. A thermal conductor (measuring probe) which encloses the platinum resistor is attached directly to PCB and has a screw thread which is intended to install ITWS in the pipes of the heating system. This design provides the best thermal contact between ITWS and the heat-conveying liquid temperature of which is being measured. The initialization and deinitialization are carried out by the magnetically-operated sealed switch. This operation is controlled by the software. The specifications of ITWS are shown in Table I.

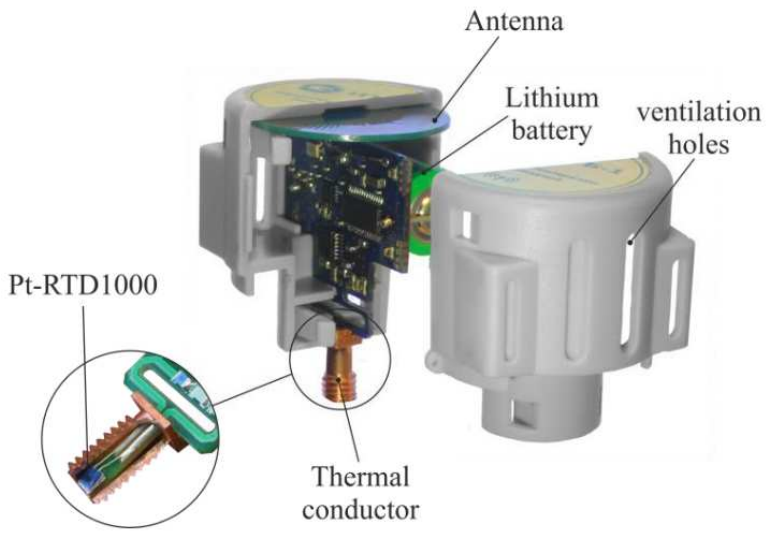

Fig. 2. The ITWS design.

The ITWS has passed tests and has been certified by Federal Agency on Technical Regulation and Metrology (Rosstandart). The ITWS also has been patented.
TABLE I

The specifications of ITWS.

\begin{tabular}{c|c}
\hline \hline Parameter & Value \\
\hline Temperature measuring range $\left[{ }^{\circ} \mathrm{C}\right]$ & $5 \div 95$ \\
Accuracy $\left[{ }^{\circ} \mathrm{C}\right]$ & \pm 0.05 \\
Thermal inertia $\varepsilon_{\infty}[\mathrm{s}]$ & 30 \\
Measuring current $[\mathrm{mA}]$ & 1 \\
Data transferring/receiving current $[\mathrm{mA}]$ & 22 \\
Frequency bands $[\mathrm{MHz}]$ & $434 / 868$ \\
Output power of the transmitter $[\mathrm{mW}]$ & 10 \\
Dimensions $[\mathrm{mm}]$ & $39 \times 39 \times 53$ \\
Weight $[\mathrm{g}]$ & 50 \\
Supply voltage $[\mathrm{V}]$ & 3.6
\end{tabular}

\section{A transfer function}

The accuracy of the electronic thermometer is mainly determined by a transfer function which describes the temperature dependence of the thermometric parameter of the sensor [2]. To develop the transfer function of ITWS, the following boundary conditions were taken into account: operating temperature range; the maximum permissible error and the minimum number of computational operations. Conducted analyses and simulations of thin-film platinum resistors permitted the authors to determine the optimal transfer function for ITWS, which provides a calculation error of $\pm 0.005^{\circ} \mathrm{C}$ in the range of 0 to $100^{\circ} \mathrm{C}$ :

$$
t=\left(W_{r}+a\right) /\left(b W_{r}+c\right),
$$

where $W_{r}$ is a ratio of the resistance $R_{t}$ at the temperature $t$ and the resistance $R_{0}$ at $0{ }^{\circ} \mathrm{C}\left(W=R_{t} / R_{0}\right)$; $a, b, c$ - coefficients which are estimated by the leastsquares method from RTD standards' tables (for instance DIN 43760) for each type of platinum sensor.

\section{Calibration procedure of ITWS}

The high requirements on the accuracy of temperature measurement cannot be satisfied without individual calibration of the ITWS during which the transfer function is adjusted for each sensor. The minimum number of calibration points is dependent on the selected technique for adjustment of the transfer function. In practice, there are two different techniques: calculation of all the coefficients in the transfer function or use of the deviation function. To describe our transfer function, we used a linear deviation function as follows:

$$
W_{r}=k W(t)+m,
$$

where $k$ and $m$ are calibration factors, $W(t)$ - the resistance ratio of the calibrated thin film platinum resistor. It is obvious that two calibration points are needed to find the calibration factors for the this function.

For the purpose of ITWS study and calibration, authors have developed an automated measurement system, the scheme of which is shown in Fig. 3. This system includes a thermostat Lauda PROLINE RP 3530 in which a calibration cell is installed. This cell can contain up to 56 ITWS for simultaneous calibration. 


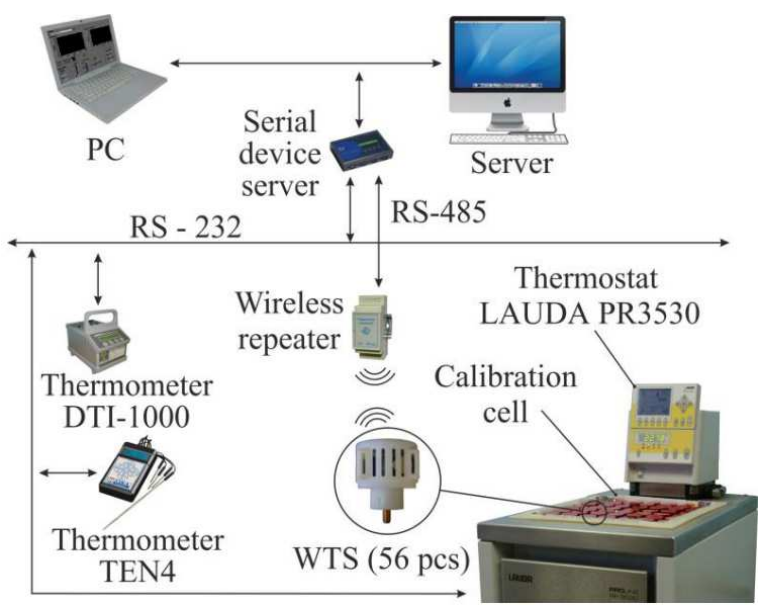

Fig. 3. The scheme of the automated measurement system for calibration of the ITWS.

To provide isothermal conditions during the calibration process, the cell was fabricated from copper slab (25 $\mathrm{mm}$ thick). The reference temperature probe is installed in the middle of the calibration cell. This probe is connected to a high-accuracy digital thermometer DTI1000 which provides measurements for the calibration process. Four platinum sensors, which are connected to a digital thermometer TEN-4 and are installed around the cell's perimeter, control the temperature profile of the calibration cell. Studies have shown that the temperature difference between these sensors is no more than $0.02{ }^{\circ} \mathrm{C}$ during the calibration process. The verification of the reference temperature probe and the platinum sensors is carried out by using ITS-90 fixed points (the triple point of water and freezing point of indium). The wireless repeater is also included in the measurements system to provide connection between calibrated ITWS and PC.

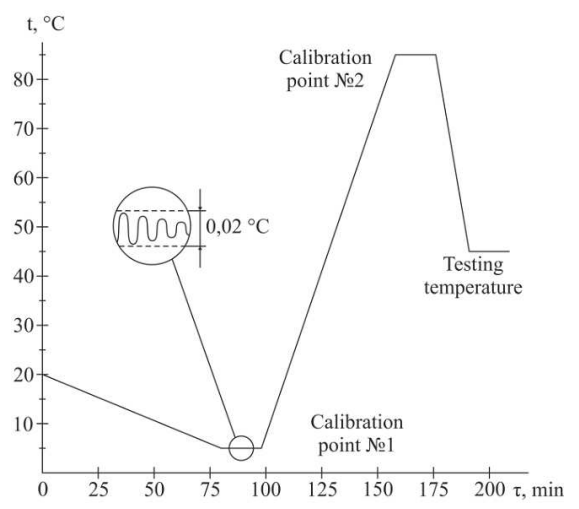

Fig. 4. The Diagram of the ITWS calibration process.

The calibration process is carried out in the sequence which is shown in the diagram in Fig. 4. The first calibration point, which is equal to $5^{\circ} \mathrm{C}$, is set by a proprietary calibration software. The DTI-1000 thermometer measures the temperature of the coolant which stabilizes within 50-70 minutes. The temperature is stabilized when it does not change by more than $0.02{ }^{\circ} \mathrm{C}$ for at least
4 minutes. After that, all ITWS are initialized and the differences between ITWS and DTI-1000 measurements are calculated and accumulated. After calibration at the temperature of $5{ }^{\circ} \mathrm{C}$, the stability of ITWS measurements is tested for the next 8 minutes.

The temperature of the next calibration point is equal to $85^{\circ} \mathrm{C}$. When this process, which is identical to that at $5{ }^{\circ} \mathrm{C}$, is completed, the coefficients of the transfer function are calculated for each of the 56 ITWS. Then the testing temperature of $45^{\circ} \mathrm{C}$ is set to compare the ITWS and DTI-1000 measured data. If ITWS measurements differ from those of DTI-1000 no more than by $\pm 0.05^{\circ} \mathrm{C}$, it is considered that ITWS is calibrated. And if this difference is more than $\pm 0.05^{\circ} \mathrm{C}$, the ITWS is considered broken.

Conducted studies of 2000 calibrated ITWS showed that their accuracy is no worse than $\pm 0.05^{\circ} \mathrm{C}$. It should be noted that the calibration software allows controlling of up to 6 Lauda PROLINE RP 3530 and simultaneously calibrating 336 ITWS.

\section{Conclusions}

A new intellectual temperature sensor with wireless communication interface has been developed. The ITWS is intended for contact measurement with an accuracy of $\pm 0.05^{\circ} \mathrm{C}$ in an operation range of 5 to $95^{\circ} \mathrm{C}$. The ITWS can be used for contact temperature measurement of any non-corrosive medium.

The proposed ITWS design is intended for use in energy consumption metering systems to measure the temperature of the heat-conveying liquid in the heating system in order to determine individual heat consumption. The measured data is transmitted from ITWS to PC over a wireless repeater (frequency bands 434 or $868 \mathrm{MHz}$, output power of the transmitter is no more than $10 \mathrm{~mW}$ ).

The high-accuracy of ITWS is ensured as follows. A transfer function which allows calculating temperature with an error of no more than $5 \times 10^{-3}{ }^{\circ} \mathrm{C}$ has been proposed. ITWS also has been patented. Original patented design, electric circuit and software were developed for ITWS. A procedure and an automated measurement system for calibration of ITWS have been proposed.

ITWS has passed tests and has been certified by Federal Agency on Technical Regulation and Metrology (Rosstandart).

\section{Acknowledgments}

This work has been carried out under a sponsorship of the Ministry of Education and Science of the Russian Federation, project number 14.575.21.0013 (UN RFMEFI57514X0013).

\section{References}

[1] Yu.I. Shtern, Ya.S. Kozhevnikov, V.A. Medvedev, R.E. Mironov, I.S. Karavaev, Meas. Tech. 56, 178 (2013).

[2] Yu.I. Shtern, Ya.S. Kozhevnikov, V.M. Rykov, R.E. Mironov, Russ. Microelectron. 42, 384 (2013). 